\title{
Memory test and repair technique for SoC based devices
}

\author{
Mohammed Altaf Ahmed ${ }^{1, \text { a), b) }}$, Abubaker E.M. Eljialy ${ }^{2}$, and Sultan Ahmad ${ }^{3}$
}

\begin{abstract}
System on Chip (SoC) architecture mainly consists of the memories in a larger area. Due to the availability of memories in a larger-size, it is difficult to test these memories for faults. Therefore, a smooth test solution to test these memories against fault and repair the faulty cells has introduced. In this research, we proposed a Memory Test Controller (MTC) to test the memories and Built-in Self-repair (BISR) mechanism to repair the faulty cells for any recent SoC based devices. The MTC not only identifies the fault, but it finds the type of fault available, and BISR block repairs the detected faulty cells. The paper provides empirical insights about how change is brought in features of the SoC based device after integrating both the proposed controller block. It is noticed that from the obtained results, the proposed methods are stands better in terms of the area overhead, power and timing when compared with the existing approaches.

Keywords: MTC, faults in memory, fault types detection, SoC, BISR

Classification: Integrated circuits (memory, logic, analog, RF, sensor)
\end{abstract}

\section{Introduction}

The current SoC based device needs many tasks to perform to sustain a reputation in the market. Among the various functions, the user wants one of the essential functions that the device must contain large memory space. The increase in the ample storage of memory in SoC design impacts its performance. More storage will expose prone to a fault [1, 2]. It results in difficulties in testing memories in terms of failures $[3,4]$. As the survey reference to report of ITRS (International Technological Roadmap for Semiconductor) conducted by Semiconductor Industry Association (SIA), the portion of memory in SoC is above $95 \%$ of the total area [5]. Thus, testing of such a dense memory in SoC has become critical [6]. Therefore, to provide the test solution for memories in any SoC-based device (for example, the latest mobile phone) is the research objective of this paper.

Due to the large size and massive amount of data storage, the memories in SoC considerably impact on the yield of the production of the devices [7]. Therefore, a useful test solu-

${ }^{1}$ Department of Computer Engineering, College of Computer Engineering and Sciences, Prince Sattam bin Abdulaziz University, Saudi Arabia

2 Department of Information System, College of Computer Engineering and Sciences, Prince Sattam bin Abdulaziz University, Saudi Arabia

${ }^{3}$ Department of Computer Science, College of Computer Engineering and Sciences, Prince Sattam bin Abdulaziz University, Saudi Arabia

a) m.altaf@psau.edu.sa

b) altaface1@gmail.com

DOI: 10.1587/elex.18.20210092

Received February 21, 2021

Accepted March 22, 2021

Publicized March 31, 2021

Copyedited April 25, 2021 tion with a fault repair mechanism will support improving the recent SoC-based systems' yield.

This research consists of two methods, first to test the memories and second one to repair the memories for fault. There are existing approaches available to test the memories for failures but can be further enhanced to get better results $[8,9,10,11,12,13,14]$. Among the various test techniques of memories, the algorithmic test approach retained its positions for a long time [15]. The algorithmic approach is fast and provides better fault coverage [9]. The stuck-at fault, transition fault, address decoder faults, neighbourhood pattern sensitive faults (NPSF), and coupling faults computes by memory built-in self-test (MBIST) approach $[9,12,16,17,18,19,20]$. The research approaches $[21,22,23]$ are not able to detect all fault types in semiconductor memories. They identify only stack-at fault, address decoder faults, transition faults, and only some coupling faults. The coupling faults are detected using a Marchee algorithm in the research [12].

Memory repair is a standard method for yield improvement for today's modern SoC design. This process usually consists of memory test, redundancy analysis (RA) and repair mechanism. The repair signature is produced by the redundancy analysis depending on the failure information received from the MTC, also known as MBIST. The BISR block uses this repair signature to repair the memory under test (MUT) [24, 25, 26, 27, 28]. Memories are more proven to faults; thus, memory testing involves various test techniques on different test algorithms and various faults modules. We cannot merely ignore the defects if they exist in memory. Therefore, the spare memory method is used. In the spare memory scheme, some predefine spare rows and spare column are reserved, and when faulty cells are going to operate, spare rows and spare columns will use in place of the defected cells by the BISR block. Therefore the MBIST, together with BISR blocks, plays a significant role in SoC development. Recent SoC based devices (for example, mobile phone) are designed with large memory size. Therefore the significant memory test method improves the yield of the products.

With the advancements in the technology of the mobile industry, the device has to have smooth performance and functioning at the hardware level. Therefore, it is required to improve the architecture of the SoC for the current generation devices. This improvement in the SoC design will ensure the smooth operation of devices towards the valueadded components in it. We targeted in this research, one of the value-added elements of ample memory storage in the SoC. A smooth memory test controller with a fault repair 
mechanism is proposed to test and repair the memories under SoC. This memory test engine examines the memories for faults and computes all the possibly generated defects and their types. The BISR block gets failure information of the memory from MTC and repairs the faulty cells by the proposed method. The proposed MTC and BISR are developed to integrate with the SoC design, and which results in a small hardware overhead. It became significant when it compares with the memory controllers available in SoC [3, 25, 28] and the BISR block [24, 26, 27, 29] of the existing approaches. The proposed MTC and BISR blocks stand better in terms of area overhead and power consumption to test and repair the memories under SoC when compared with existing methods. For example, it takes only $2.5 \%$ additional hardware overhead in case of MTC and 3.1\% in case of BISR with 8kx64 memory size.

The contribution of this research work listed as,

- Proposing a memory test controller (MTC) based on modified March $\mathrm{C}$ algorithm to test memories for faults and their types in the modern SoC.

- Presenting the BISR method to repair the faults for MUT depending on the fault signature of BIRA.

- Comparison of the finding of the proposed method with other existing approaches in terms of area, power and timing enhancement.

The rest of the paper is structured as section 2 consists of the architecture of the proposed MTC and BISR controller. Section 3 consists of the experimental results and comparison with the existing methods. Section 4 outlines the conclusion and future scope.

\section{Proposed architecture of MTC and BISR}

The proposed architecture of an SoC-based device is consists of two central part first one is MTC, and the second one is BISR, as shown in Fig. 1. The MTC and BISR controllers are used to integrate into the $\mathrm{SoC}$ design to enhance the performance and features of a device.

\subsection{Memory test controller (MTC)}

The MTC have six main blocks, two register unit to start the state machine and to record the fail information, a controller state machine on modified March $\mathrm{C}$ algorithm, a test pattern generator, a comparator to compare the output with

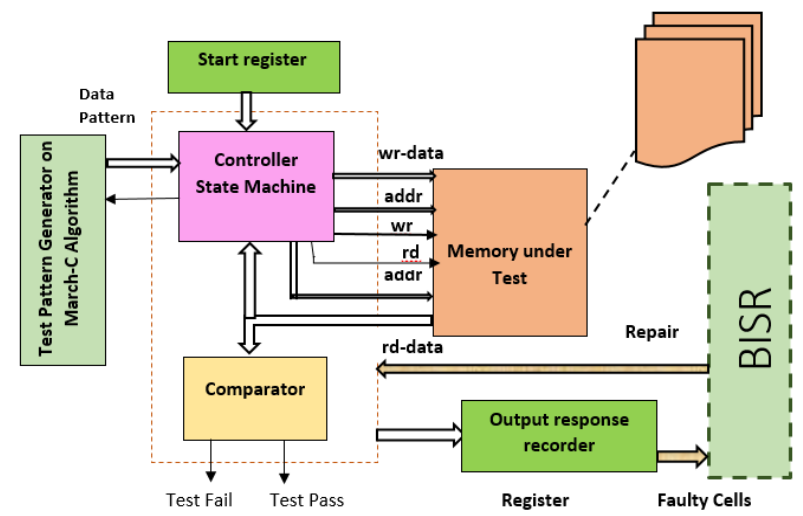

Fig. 1 The architecture of the memory test controller and repair logic for an SoC based embedded device. the desired pattern and a memory under test (MUT) block. The start register consists of clock, reset, start, resume, stop, memory id (two bits), and halt-on-error. The state machine begins once the start signal is programmed in start register. It triggers the state machine to perform the operations mentioned in the algorithm.

The test pattern generator will work on the proposed modified March $\mathrm{C}$ algorithm and generates the patterns as specified in the algorithm. These generated patterns are applied to the memory under test, during the read operations, while testing, read data compared with the desired patterns if the comparison fails, the state machine jumps to the status-offail-record state and records the comparison results. The memory ID, fail address, faulty cells, and defective cell count of the MUT records in the output response recorder.

These fail information is transfer to the BISR block to use spare memory instead of the faulty cells.

\subsubsection{Test algorithm}

The memory test controller works on the modified form of March $\mathrm{C}$ algorithm to improve fault coverage. We modified March $\mathrm{C}$ element because it is not able to detect coupling faults. The predefine patterns applied to the memory under test (MUT), and the responses recorded into the output response recorder. The writing patterns into the memory and simultaneously reading them from memory which described in the algorithm, the state machine is designed. The state machine consists of the state equal to the operations specified in the algorithm, and also two more states, idle and status-of-fail-record. The purpose of an idle state is to start operations, and the status-of-fail-record state is to store the fail information. In the present research, we proposed the modified March $\mathrm{C}$ algorithm for conducting the test, which has given below.

$\frac{\text { Proposed Modified March-C algorithm }}{\frac{\mathrm{E}^{1}: \uparrow \downarrow(w 0) ; \mathrm{E}^{2}: \uparrow(\mathrm{r} 0, \mathrm{w} 1) ; \mathrm{E}^{3}: \uparrow(\mathrm{r} 1, \mathrm{w} 0, \mathrm{r} 0) ;}{\mathrm{E}^{4}: \downarrow(w 0, \mathrm{r} 0, w 1) ; \mathrm{E}^{5}: \downarrow(\mathrm{r} 1, \mathrm{w} 0) ; \mathrm{E}^{6}: \downarrow(\mathrm{r} 0) ;}}$

Steps in the algorithm specified in Table I, further the operation sequence in each step explained below. There are two modified march elements $\mathrm{E}^{3}$ and $\mathrm{E}^{4}$ in the algorithm as compared with March C [30], and MTC works as per these steps. At each step, the operations under the parenthesis (read/write) are performed sequentially at each memory location (in ascending order or descending order as mentioned in the algorithm).

\subsubsection{State machine}

The state machine designed for the memory controller that consists of eight states, and it works on modified March C

Table I Illustrations used in the algorithm

\begin{tabular}{l|l}
\hline Operation & Description \\
\hline$\uparrow \downarrow$ & $\begin{array}{l}\text { This notation of modified March C indicates that the address } \\
\text { ordering of the memory is not essential, ascending (from zero to } \\
\text { maximum).or descending (from maximum to zero). }\end{array}$ \\
\hline$\uparrow$ & The address order of the memory has indicated in ascending order. \\
\hline$\downarrow$ & The address order of the memory has indicated in descending. \\
\hline w0 & Write 0s at the memory location. \\
\hline w1 & Write 1s at the memory location. \\
\hline r0 & The memory location, whose value is 0, read the data. \\
\hline $\mathrm{r} 1$ & The memory location, whose value is 1, read the data. \\
\hline
\end{tabular}




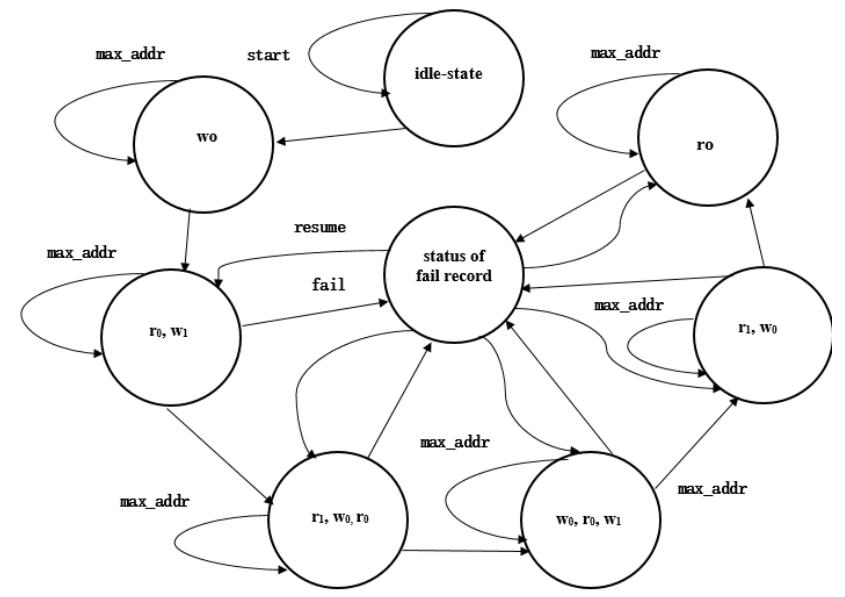

Fig. 2 Memory controller state machine

principles shown in Fig. 2.

These states are listed as follows:

(1) Idle-state (2) w0 (3) r0w1 (4) r1w0r0 (5) w0r0w1

(6) r1w0 (7) r0 (8) status-of-fail-record

The state machine in idle state wait for the start signal once received it jumps to the w0 state and starts memorytest operation as described in the algorithm respectively. The memory under test is full of zero patterns when it jumps to the next $\mathrm{r} 0 \mathrm{w} 1$ state. In this state, the state machine will perform two operations first read and then write as described in the algorithm. Similarly, the state machine performs all these operations sequentially at each state. While testing, read data compared with the desired patterns during the read operations. If the comparison fails, the state machine jumps to the status-of-fail-record state. It records the comparison results and resumes back to the next address location from where it arrived. The memory ID, fail address, faulty cells, and defective cell count of the MUT records in the output response recorder. In this manner, it completes all operations and displays the result in the status-of-fail-record state. The state machine can detect the fail memory ID, location of faults, faulty cell and faulty cell count. Moreover, the simulation process computes various fault types. This research methodology determines the stuck-at (SA) fault, address decoder fault, transition fault, and coupling faults.

\subsection{Built in self repair}

The memory fails information (memory ID, faulty location, faulty cell and faulty cell count) from the status of fail record register is transfer to the BISR block to repair of the defective cells of the fail memory. The BISR block consists of redundancy logic (RL) and the spare memory (row-column) block and is shown in Fig. 3. The RL block is used to store faulty addresses found during the memory test process. During read and write operation of memory, the address is compared with the addresses available in the redundancy logic. If it matches, the BISR will work, and data will access through the spare memory. The BIRA block accesses the failure information, calculates the repair signature, and stores it in the BIRA register. It also compares the defected addresses with the previously saved addresses in the fault table in case of multiple faults. The address is stored only when if it is not available in the fault table. BIRA will decide the spare

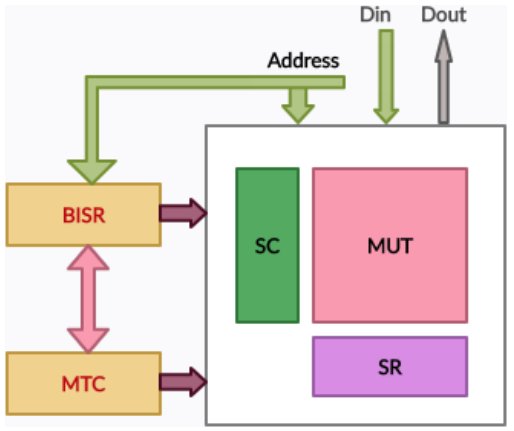

Fig. 3 BISR block diagram

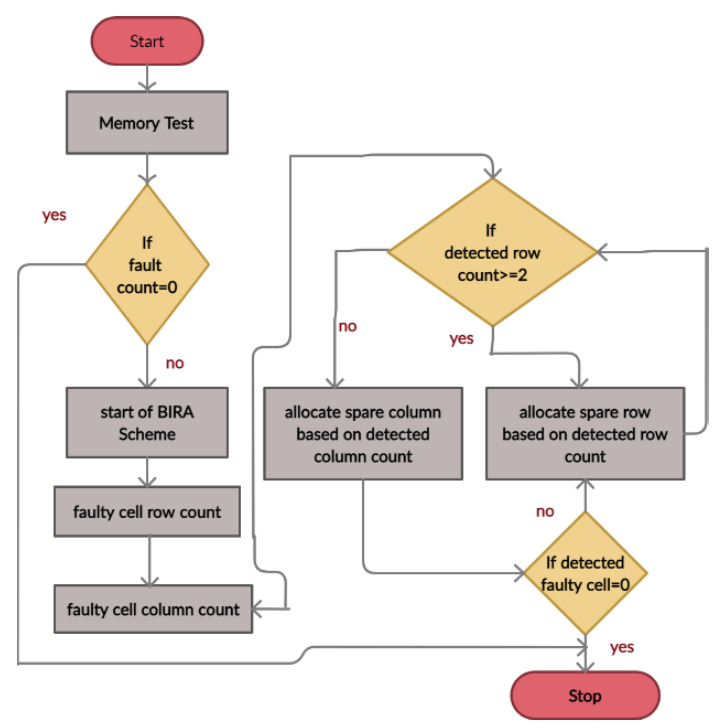

Fig. 4 BISR algorithm flow

row/column allocation based on the faulty cell count information in a particular address. The BISR block works on the principle shown in the flow chart of Fig. 4.

The flow chart steps are as follows:

1. The repair principle works on the simple rule, if a row has multiple faults, it will repair, and if the column has numerous faults at different row, the column will fix.

2. The faulty cell count is received by MTC and is taken as a reference by BIRA to calculate the repair signature.

3. A predefined threshold value to use spare rows and spare columns will decide depending on the count of defected cells in defected rows or defected columns. The predefined threshold value is equal to or greater then ' 2 '.

4. If row defect count is greater or equal to ' 2 ', the spare row will allocate first than the column; otherwise, the spare column will allocate first.

5. Once the spare memory (row/column) allocation finished, the remaining defected cells' checking process will carry on. If it is non zero, the spare row will allocate, the checking process continuing until it reaches null.

6. The spare memory can be increase depending on the faulty cells count. However, we considered in this research two spare rows and two spare columns. 


\section{Results and comparison}

The memory controller and repair mechanism for any SoCbased device has developed on ASIC (Application Specific Integrated Circuit) by Cadence tool Encounter(R) RTL Compiler with $180 \mathrm{~nm}^{2}$ technology. The RTL (Register Transfer Logic) code has written in Verilog HDL (Hardware Description Language). The simulation has conducted on the Xilinx simulator. Therefore, the experiments yield through two steps functional verification and logic verification.

\subsection{Functional verification}

The step involves, the verification of design for the functional correctness of both the blocks MTC and BISR, which are implemented in Verilog HDL. The Xilinx ISE tool performs a functional check. In this step, we can compute the damaged cells, and the type of fault exists in a particular memory at a specific address. The verification environment has formed to test the embedded memories in an SoC. The test cases are written to detecting the faults and finding damaged cells from memory under test. The data patterns are written to compute the faults, and their types are shown in Table II of Case I, Case II, Case III, and Case IV.

Case I: In this case, the SA-1 fault computes by writing zero patterns. The location 1, detects a SA-1 failure at 1st-bit position and location 3 at 29th and 30th-bit position.

Case II: The SA-0 fault computes by performing procedures of algorithm in this state. The SA-0 error detected at the location 2 position one and the location 5 at place 19 .

Case III: In this case, the state machine computes the transition faults and address decoder faults. The address decoder faults are present at memory location 15 and 7 . The transition faults appear at location 3 and location 6 of MUT.

Case IV: The coupling fault appears when the value of cell changes due to couple with another cell. In this case, the coupling fault presents at location 6 on cell position 56, and it coupled with another cell 22 of memory location 14 . Therefore, the value of cell 56 at the sixth location force to change the value of cell 22 at location 14. Similarly, cell

Table II Fault and fault type detection

\begin{tabular}{|c|c|c|}
\hline Address & Data Pattern & Fault exist \\
\hline \multicolumn{3}{|c|}{ Case I Stuck-at-1 Faults } \\
\hline 0 & 0000000000000000 & \\
\hline 1 & 0000000000000001 & Stuck-at-1 fault \\
\hline 3 & 0000000030000000 & Stuck-at-1 fault \\
\hline \multicolumn{3}{|c|}{ Case II Stuck-at-0 Faults } \\
\hline 2 & ffff ffff ffff fffe & Stuck-at- 0 fault at 1 \\
\hline 5 & ffff ffff fffb ffff & Stuck-at-0 fault at 19 \\
\hline \multicolumn{3}{|c|}{ Case III transition faults and address decoder faults } \\
\hline 2 & 0000000000000000 & \\
\hline 3 & 0000000000004000 & Transition fault at 15 \\
\hline 4 & ffff ffff ffff ffff & Address Decoder fault \\
\hline 6 & ffff ffff fffb ffff & Transition fault at 19 \\
\hline 7 & 0000000000000000 & Address Decoder fault \\
\hline 15 & ffff ffff ffff ffff & \\
\hline 699 & ffff ffff ffff ffff & Address Decoder fault \\
\hline \multicolumn{3}{|c|}{ Case IV coupling faults } \\
\hline 5 & 0000000000000000 & \\
\hline 6 & 0000000000000000 & Coupled with cell 22 at location 14 \\
\hline 8 & 0000000000000000 & Coupled with cell 4 at location 13 \\
\hline 13 & ffff ffff ffff fff7 & Coupling fault at cell 4 \\
\hline 14 & ffff ffff ffdf ffff & Cell 22 Coupled with a cell 56 of location 6 \\
\hline
\end{tabular}

number ten at location 8 , coupled with cell number 4 at location 13 , due to coupling fault.

Simulation results for the functional verification and the fault type detection for the proposed controller is shown in Fig. 5. This simulation waveform represents the writing and reading of the data patterns into the MUT. The writing and reading operations performed, as mentioned in the algorithm. The SA fault, transition fault, coupling fault, and address decoder faults are computed and are written in the fault table for BIRA.

\subsection{Logic verifications}

Both MTC and BISR, are developed on ASIC (Application Specific Integrated Circuit) for any SoC-based device. A synthesis process carried out on Cadence Synthesizer Encounter(R) RTL Compiler with the product version v11.10p005_1 and $180 \mathrm{~nm}^{2}$ technology. The synthesis results for both the design are obtained in terms of the area and power consumption.

\subsubsection{ASIC results for MTC}

The total cell count, cell area, net area and the total area received for the MTC is shown in Table III. Similarly, other parameters, like delay and power consumption, are also computed by ASIC synthesis. The timing (delay) obtained by the synthesis result is $2376 \mathrm{ps}$, and the power consumption by the MTC design is tabulated in Table IV.

In this research, the experiments have conducted on
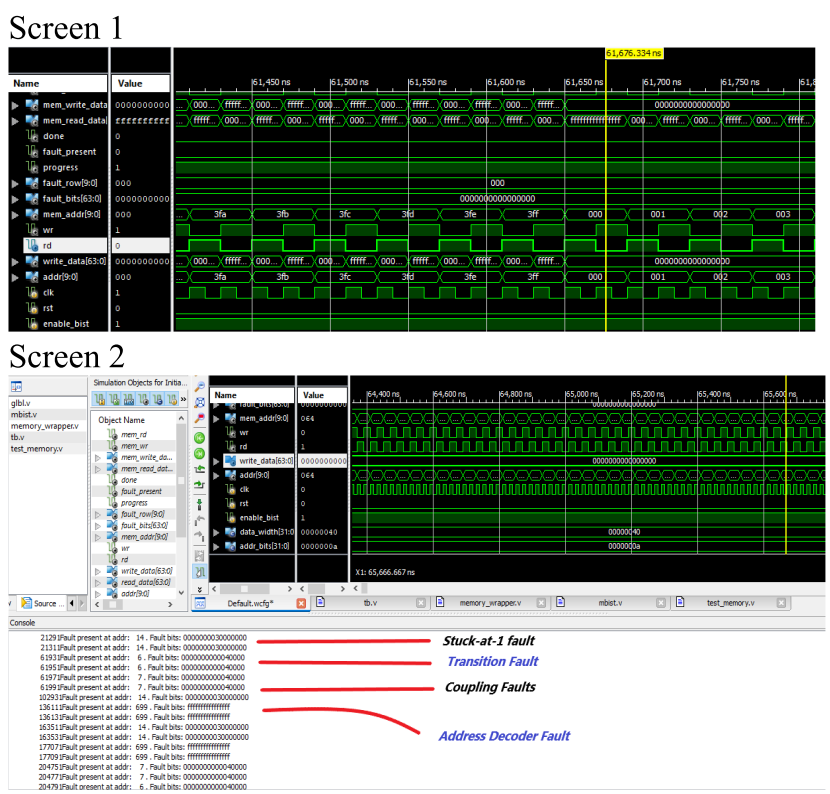

Fig. 5 Simulation results for faults and fault type detection

Table III Memory controller area by ASIC synthesis

\begin{tabular}{l|l|l|l|l}
\hline Design & Cell & $\begin{array}{l}\text { Cell Area } \\
(\mathrm{nm} 2)\end{array}$ & $\begin{array}{l}\text { Net Area } \\
(\mathrm{nm} 2)\end{array}$ & $\begin{array}{l}\text { Total Area } \\
(\mathrm{nm} 2)\end{array}$ \\
\hline $\begin{array}{l}\text { Memory Controller on } \\
\text { Modified March-C }\end{array}$ & 579 & 6324 & 3869 & 10071 \\
\hline
\end{tabular}

Table IV Power consumption

\begin{tabular}{l|l|l|l|l}
\hline \multirow{2}{*}{ Instance } & \multicolumn{4}{|l}{ Power $(\mathrm{nW})$} \\
\cline { 2 - 5 } & Cell & Leakage & Dynamic & Total \\
\hline Proposed MTC & 579 & 2007.871 & 1193227.532 & 1191245.204 \\
\hline
\end{tabular}




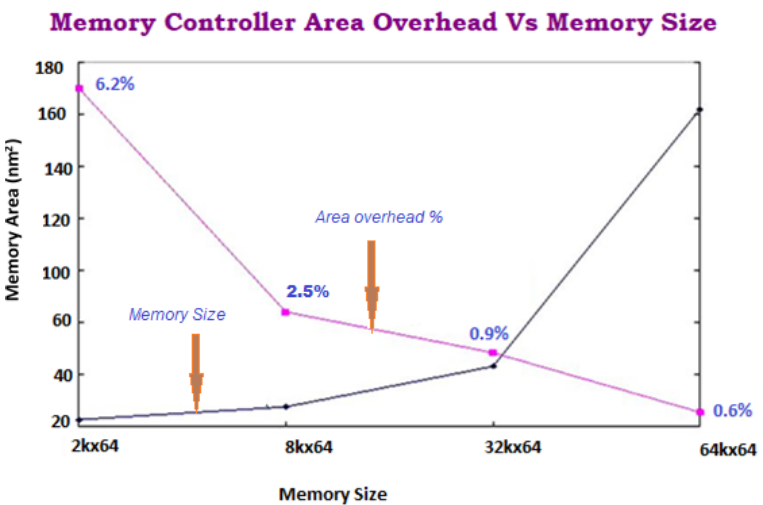

Fig. 6 Memory controller area overhead vs memory size plot

Table V Memory size versus area overhead of MTC

\begin{tabular}{l|l|l|l}
\hline Memory Size & $\begin{array}{l}\text { Memory Area } \\
(\mathrm{nm} 2)\end{array}$ & $\begin{array}{l}\text { Memory Test } \\
\text { Controller Area }(\mathrm{nm} 2)\end{array}$ & $\begin{array}{l}\text { Area Overhead } \\
(\%)\end{array}$ \\
\hline $2 \mathrm{kx} 64$ & 142255 & 8910 & 6.26 \\
\hline $4 \mathrm{kx} 64$ & 227608 & 8956 & 3.93 \\
\hline $8 \mathrm{k} \times 64$ & 396719 & 10071 & 2.5 \\
\hline $16 \mathrm{Kx} 64$ & 582678 & 9087 & 1.55 \\
\hline $32 \mathrm{kx} 64$ & 932284 & 9113 & 0.977 \\
\hline $64 \mathrm{kx} 64$ & 1491654 & 9233 & 0.618 \\
\hline
\end{tabular}

Table VI Area overhead comparison of proposed and existing approaches

\begin{tabular}{l|l|l|l|l}
\hline \multirow{2}{*}{$\begin{array}{l}\text { Memory } \\
\text { Size }\end{array}$} & \multicolumn{4}{|c}{ Area Overhead (\%) } \\
\cline { 2 - 5 } & $\begin{array}{l}\text { Chin-Lung } \\
\text { et al. [28] }\end{array}$ & $\begin{array}{l}\text { Chang, DM } \\
\text { et al. [3] }\end{array}$ & $\begin{array}{l}\text { Xiao-feng } \\
\text { et al. [25] }\end{array}$ & Proposed MTC \\
\hline 2kx64 & 10.6 & 9.1 & 7.8 & 6.26 \\
\hline $4 \mathrm{kx} 64$ & 6.71 & 5.7 & 4.2 & 3.93 \\
\hline $8 \mathrm{kx} 64$ & 4.1 & 3.5 & 3.2 & 2.5 \\
\hline $16 \mathrm{Kx} 64$ & 2.64 & 2.26 & 2.8 & 1.55 \\
\hline $32 \mathrm{kx} 64$ & 1.66 & 1.42 & 1.1 & 0.977 \\
\hline $64 \mathrm{kx} 64$ & 1.05 & 0.9 & 0.8 & 0.618 \\
\hline
\end{tabular}

8Kx64 memory. The total area received for the $8 \mathrm{Kx} 64$ memory is $396719 \mathrm{~nm}^{2}$, and for the MTC obtained area is $10071 \mathrm{~nm}^{2}$, which is approximately $2.5 \%$ of the $8 \mathrm{k}$ memory area.

Therefore, the area overhead approximately $2.5 \%$ is received. The area of the memory controller always fixed with the different sizes of the memory. Consequently, it can conclude as memory size increases, the area overhead of the memory controller decreases in any SoC-based device. Different memory sizes with the same memory controller affect area overhead as given in Table $\mathrm{V}$ and illustrated in the graph (Fig. 6). The area overhead comparison of the proposed MTC with different existing methods is given in the Table VI.

\subsubsection{ASIC results for BISR}

Similarly, the BISR block is also implemented on ASIC, and the results have obtained for area, power and timing. The obtained results are compared with the existing methods of BISR. The existing methods of the research [24, 27, 31] are not enough to test the memory for fault, they either use fault injection method for getting faulty cells information instead of MTC or just comparing neighbouring cells to assume defects in the memory. The comparison with different existing approaches of BISR is given in Table VII.

The area report of the proposed BISR consists of 482 as a total cells count, and the entire area required for the BISR block is received as $8636 \mathrm{~nm}^{2}$ and is compared with the area report of the said existing methods. The comparison is
Table VII Features wise comparison BISR approaches

\begin{tabular}{l|l|l|l|l}
\hline & $\begin{array}{l}\text { Cresta } \\
{[27]}\end{array}$ & $\begin{array}{l}\text { Counting } \\
\text { Threshold [31] }\end{array}$ & $\begin{array}{l}\text { paralogous gene } \\
{[24]}\end{array}$ & $\begin{array}{l}\text { Propos } \\
\text { ed }\end{array}$ \\
\hline $\begin{array}{l}\text { Fault Detection } \\
\text { support (MTC) }\end{array}$ & No & No & No & Yes \\
\hline Spare rows & 3 & 3 & 16 Cell & 2 \\
\hline Spare columns & 3 & 1 & 16 Cell & 2 \\
\hline $\begin{array}{l}\text { Fault Detection } \\
\text { Method }\end{array}$ & $\begin{array}{l}\text { Fault } \\
\text { insertion }\end{array}$ & $\begin{array}{l}\text { Fault } \\
\text { insertion }\end{array}$ & $\begin{array}{l}\text { Neighboring cell- } \\
\text { based decision }\end{array}$ & MTC \\
\hline
\end{tabular}

Table VIII Area report of the proposed BISR block

\begin{tabular}{l|l|l|l|l}
\hline Design & Cell & $\begin{array}{l}\text { Cell Area } \\
(\mathrm{nm} 2)\end{array}$ & $\begin{array}{l}\text { Net Area } \\
(\mathrm{nm} 2)\end{array}$ & $\begin{array}{l}\text { Total Area } \\
(\mathrm{nm} 2)\end{array}$ \\
\hline Proposed BISR & 482 & 5876 & 2968 & 8636 \\
\hline Counting Threshold [31] & 596 & 6036 & 3978 & 10023 \\
\hline Paralogous gene [24] & 894 & 10437 & 5870 & 15392 \\
\hline Cresta [27] & 908 & 11036 & 6013 & 16972 \\
\hline
\end{tabular}

Table IX Area overhead, timing, and power comparison of BISR

\begin{tabular}{l|l|l|l|l}
\hline & Area & Power & Area overhead & Timing \\
\hline Cresta [27] & High & Average & High & Moderate \\
\hline paralogous gene [24] & High & High & High & Fast \\
\hline Counting Threshold [31] & Average & Average & Moderate & Moderate \\
\hline Proposed BISR & Low & Low & Low & Fast \\
\hline
\end{tabular}

Table X Area overhead calculation for both the blocks

\begin{tabular}{l|l}
\hline MTC block obtained area & BISR block obtained area \\
\hline Memory Controller area- $10071 \mathrm{~nm}^{2}$ & BISR area $=8636 \mathrm{~nm}^{2}$ \\
Memory area- $396719 \mathrm{~nm}^{2}$ & Memory area $=396719 \mathrm{~nm}^{2}$ \\
Area overhead $=2.5 \%$ & Spare Memory area $=4028 \mathrm{~nm}^{2}$ \\
& Area overhead $=3.19 \%$ \\
\hline
\end{tabular}

tabulated, in Table VIII.

The power consumption and the timing have also computed for the presented BISR block. They are relatively compared with the obtained power, area, and timing results of the existing approaches, given in Table IX. The total memory (8kx64) area received is $396719 \mathrm{~nm}^{2}$. It is the same as in the case of MTC. The obtained BISR area and spare memory area is $8636 \mathrm{~nm}^{2}$, and $4028 \mathrm{~nm}^{2}$ respectively, which is approximately $3.19 \%$ of the $8 \mathrm{k}$ memory area, and it is given in the Table X.

It has noticed that the proposed methods of the MTC and BISR are more efficient in terms of area overhead, power, and timing when compared with the existing techniques of memory testing and self-repair. The area overhead is only a limitation of this proposal, but it cannot ignore.

\section{Conclusion}

Memory test and repair technique has implemented for SoC based devices. The functional and logical test has carried out. The functional analysis computes the availability of faults and their types in memory, which is under examination. The logic synthesis has carried out to calculate the area overhead, timing, and power consumption. In this research, we considered an $8 \mathrm{Kx} 64$ memory to conduct the test. It obtained from the results, and the area overhead is approximately $2.5 \%$ and $3.1 \%$ for MTC and BISR respectively when they integrate with $8 \mathrm{kx} 64$ memory. It has noticed from the results that the overhead area is decreases by increasing the memory size. The only limitation of this approach is a little area penalty with it integrate to any SoC. The proposed controllers can easily modify for any future SoC design and blend at the time of manufacturing. 


\section{Acknowledgments}

This project was supported by the Deanship of Scientific Research at Prince Sattam bin Abdulaziz University under the research project \# 2019/01/11244.

\section{References}

[1] A. Bosio, et al.: Advanced Test Methods for SRAMs: Effective Solutions for Dynamic Fault Detection in Nano Scaled Technologies (Springer, 2010) 1 (DOI: 10.1007/978-1-4419-0938-1).

[2] A. Bosio, et al:: Advanced Test Methods for SRAMs: Effective Solutions for Dynamic Fault Detection in Nanoscaled Technologies (Springer-Verlag US, 2009) (DOI: 10.1007/978-1-4419-0938-1).

[3] D.-M. Chang, et al.: "A built-in redundancy-analysis scheme for random access memories with two-level redundancy," J. Electron. Test 24 (2008) 181 (DOI: 10.1007/s10836-007-5032-4).

[4] M.A. Ahmed, et al.: "FPGA based high speed memory BIST controller for embedded applications," IJST 8 (2015) 1 (DOI: 10.17485/ ijst/2015/v8i33/76080).

[5] O. Hasan, et al.: "Formal probabilistic analysis of stuck-at fault in reconfigurable memory array," 7th Int. Conf. IFM (2009) 277 (DOI: 10.1007/978-3-642-00255-7_19).

[6] M.A. Ahmed, et al.: "Embedded memory test strategies and repair," Int. J. Engg. (IJE) Trans. C: Aspects 30 (2017) 839 (DOI: 10.5829/ije. 2017.30.06c.03).

[7] X. Peng, et al.: "Optimizing weight mapping and data flow for convolutional neural networks on processing-in-mem architectures.” IEEE Trans. Circuits Syst. I, Reg. Papers 67 (2020) 1333 (DOI: 10.1109/tcsi. 2019.2958568).

[8] B. Chong and S. Zhang: "Algorithm-based fault tolerance for discrete wavelet transform implemented on GPUs," J. Sys. Arch. 108 (2020) 101823 (DOI: 10.1016/j.sysarc.2020.101823).

[9] Y. Wang, et al.: "The improvement of march C+ algorithm for embedded memory test," NCCET 2015: Computer Engineering and Technology (2016) 31 (DOI: 10.1007/978-3-662-49283-3 4).

[10] J. Kim, et al:: "Hardware-efficient built-in redundancy analysis for memory with various spares," IEEE Trans. Very Large Scale Integr. (VLSI) Syst. 25 (2017) 844 (DOI: 10.1109/tvlsi.2016.2606499).

[11] I. Mrozek and V. Yarmolik: "Pseudo-exhaustive random access memory testing based on march tests with random background variation," 2018 IEEE EWDTS (2018) (DOI: 10.1109/ewdts.2018.8524824).

[12] M.A. Ahmed and A.M. Abuagoub: "MBIST controller based on March-ee algorithm," Journal of Circuits, Systems and Computers (2020) (DOI: 10.1142/S0218126621501607).

[13] M. Koyanagi: "Recent progress in 3D integration technology," IEICE Electron. Express 12 (2015) 20152001 (DOI: 10.1587/elex.12. 20152001).

[14] E. Buslowska and V.Yarmolik: "Multi-run march tests for pattern sensitive faults in RAM," 2018 IEEE EWDTS (2018). (DOI: 10.1109/ ewdts.2018.8524643)

[15] P.K. Veenstra: Random Access Memory Testing: Theory and Practice, The Gains of Fault Modelling (Trefw.: elektronische schakelingen, Nov-2020).

[16] J.-F. Li, et al.: "Diagnostic data compression techniques for embedded memories with built-in-self-test," J. Electron. Test. Theory Appl. 18 (2002) 515 (DOI: 10.1023/A:1016557927479).

[17] C.-W. Wang, et al.: "Fault pattern oriented defect diagnosis for memories,” Proc. ITC (2003) 29 (DOI: 10.1109/TEST.2003.1270822).

[18] L.-T. Wang, et al.: in VLSI Test Principles and Architectures, ed. L.-T. Wang, et al. (Elsevier, 2006) 37 (DOI: 10.1016/ B978-012370597-6/50006-8).

[19] L.-T. Wang, et al.: VLSI Test Principles and Architectures: Design for Testability (Elsevier, 2006) (DOI: /10.1016/b978-0-12-370597-6. x5000-8).

[20] C.-W. Wang, et al.: "A built-in self-test and self-diagnosis scheme for hetero SRAM clusters," Proc. 10th ATS (2001) 103 (DOI: 10.1109/ ATS.2001.990267).

[21] G.P. Acharya and M.A. Rani: "Survey of test strategies for system- on chip and it's embedded memories," 2013 IEEE Recent Advances in Intelligent Computational Systems (RAICS) (2013) (DOI: 10.1109/raics.2013.6745473).

[22] S. Hamdioui, et al.: "A new test paradigm for semiconductor memories in the nano-era," Proc. Asian Test Symposium (2011) 347 (DOI: 10.1109/ats.2011.87)

[23] N. Li, et al.: "Logic BIST: state-of-the-art and open problems," (2015) arXiv:1503.04628v1 [cs.AR]

[24] K. Cho, et al.: "A survey of repair analysis algorithms for memories," ACM Computing Surveys 49 (2016) 47 (DOI: 10.1145/2971481).

[25] K. Cho, et al.: "An efficient built-in self-repair scheme for area reduction," ISOCC (2017) (DOI: 10.1109/isocc.2017.8368791).

[26] K. Jamal, et al.: "Test pattern generation using thermometer code counter in TPC technique for BIST implementation," Microprocess. Microsyst. 71 (2019) 102890 (DOI: 10.1016/j.micpro.2019.102890).

[27] T. Kawagoe, et al:: "A built-in self-repair analyzer (CRESTA) for embedded DRAMs," Proc. of the Int. Test Conference (2000) 567 (DOI: 10.1109/TEST.2000.894250).

[28] C.-L. Su, et al:: "A built-in self-diagnosis and repair design with fail pattern identification for memories," IEEE Trans. Very Large Scale Integr. (VLSI) Syst. 19 (2011) 2184 (DOI: 10.1109/TVLSI. 2010.2073489)

[29] K.H. Choi, et al.: "A decoupled bit shifting technique using data encoding/decoding for DRAM redundancy repair," IEICE Electron. Express 14 (2017) 20170385 (DOI: 10.1587/elex.14.20170385).

[30] M.I. Masnita, et al.: "March-based SRAM diagnostic algorithm for distinguishing stuck-at and transition faults," IEICE Electron. Express 6 (2009) 1091 (DOI: 10.1587/elex.6.1091).

[31] K. Gopalan and S. Pothiraj: "A saboteur and mutant based built-in self-test and counting threshold-based built-in self repairing mechanism for memories," Journal of Ambient Intelligence and Humanized Computing (2020) (DOI: 10.1007/s12652-020-02284-5). 